\title{
Agent-based negotiation model for long-term carpooling: a flexible mechanism for trip departure times
}

\author{
I. Hussain, L. Knapen, M. Arsalan Khan, T. Bellemans, \\ D. Janssens \& G. Wets \\ Transportation Research Institute (IMOB), Hasselt University, Belgium
}

\begin{abstract}
In order to commute by carpooling, individuals need to communicate, negotiate and coordinate, and in most cases adapt their daily schedule to enable cooperation. Through negotiation, individuals (agents) can reach complex agreements in an iterative way. The success of negotiation highly depends on the lifestyle factors that influence the departure time decision of the individuals. This paper presents a conceptual design of an agent-based model of a set of candidate carpoolers that serves as a proof of concept and is an extension of a simple negotiation model for carpooling. The proposed model extends the previous one by incorporating a more realistic departure time preference function for each agent by taking; (i) traveling, (ii) socio-economic characteristics, and (iii) time pressure factors into account for a specific activity. From the simulation's discussions, it is possible to portray the real picture of people's preferences for selecting the optimal departure time. The Janus (multi-agent) platform is used for simulating the interactions of autonomous agents with their agenda. The future research will mainly focus on incorporating different daily activities in addition to work and home activities.
\end{abstract}

Keywords: negotiation, departure time, carpooling, commuting, agent technology, Janus platform.

\section{Introduction}

Carpooling is considered to be an effective alternative transportation mode that is eco-friendly and sustainable as it enables commuters to share travel expenses, save on fuel and parking costs, improve mobility options for non-drivers and it 
also reduces emission and traffic congestion. Change in some socio-economic characteristics (SEC) such as the increase in fuel price, in parking costs, or in the implementation of a new traffic policy, may prove to be an incentive to carpool. Strict timing constraints in the schedule of the day however, have the opposite effect. In order to commute by carpooling, individuals need to communicate, negotiate and coordinate, and in most cases adapt their daily schedule to enable cooperation. Through negotiation, agents (individuals) can reach complex agreements in an iterative $[1,2]$.

While traditional modeling tools cannot handle the complexity of negotiation for carpooling, agent-based models (ABMs) are able to do so through modeling the interaction of autonomous agents. Currently many research areas including transportation behavior need to analyze and model complex interactions between different autonomous entities [3].

For the selection of trip departure time in or during the carpooling negotiation process, existing research focuses on constant preference for each individual throughout the available time window. The aim of this research is to embed the actual human behavioral preferences for trip execution during the entire departure time interval in the agent-based simulation model for long-term carpooling [4]. Thus, our model aims to extend the previous models by incorporating a more realistic departure time preference function by considering three different types of factors namely; (i) traveling (i.e. free flow travel, expected congestion, waiting and access times), (ii) SEC (i.e. ratio of travelling cost to annual income), and (iii) the time pressure i.e. the individual tolerance level for arriving late or early, into account for a specific activity.

The model is based on an agent-based and organizational meta-model [5], in which the role and organization concepts are first class entities. In the proposed conceptual model agents are the individuals, who negotiate to reach an agreement to carpool. The carpooling related actions performed by agents are divided into three phases: exploration and communication, negotiation and trip execution (carpooling). During the exploration the agent looks for other individuals to cooperate on commuting trips (home-to-work and work-to-home) during a period of multiple months. Agents explore their social network by sending requests for carpooling within a small group (who share the home and work locations with them). While negotiating, agents can reach complex agreements depending on the matching mechanism (discussed in section 3.2), used to match with preferences, which are expressed by all negotiating partners. For the trip execution, carpoolers need to coordinate with each other for the longterm carpooling. Carpoolers may (re)negotiate timing and/or (re)schedule their agenda when someone joins or leaves the carpool. The Janus [6], agent based platform is used; it provides an efficient implementation of organizational-based and agent-based concepts.

This paper is organized as follows; first we briefly describe the related work on negotiation mechanisms and carpooling models in section 2. Section 3 covers the negotiation model for the long-term carpooling. Section 4 explains the experimental setup and some results of the simulation. Finally, conclusions and future work are presented in section 5 . 


\section{Related work}

According to literature review, the agent-based models are also used in noncomputing related scientific domains and can provide valuable information on society and the outcomes of social actions or phenomena. A detailed literature review [7,8], focuses on technical development of the carpooling support systems, and empirical, interrelationships between willingness to carpool and socio-economic attributes of carpooling, is presented.

Galland et al. [1] presented a conceptual design of an ABM for the carpooling application that is used for simulating the interactions of autonomous agents and to analyze the effect of change in factors of infrastructure, behavior and cost. This model used agents' profiles and social networks to initialize communication, and a utility function to trigger the negotiation process between agents. A simple negotiation mechanism is employed with constant preference values for the entire preferred time interval. However, despite using a simple negotiation mechanism; the author conceptually sketched a methodology to formulate a behaviorally sound negotiation mechanism by defining utility function.

Hussain et al. [9] proposed a single trip negotiation model for carpooling using a simple negotiation mechanism. The first implementation used home and work locations as well as preferred trip start times and carpool periods determined by uniformly sampling given sets. Authors proposed the effective trip start time; by taking the average of preferred trip start time of each individual for the carpool. The authors extended the single-trip negotiation mechanism into a multiple trip negotiation model [4] by taking the possibility of flexible activity scheduling into account and limit the interaction between agents within small groups based on the origin and destination similarity.

Ronald et al. [10] presented an agent based model that focuses on the negotiation methodology. The proposed model includes a well-defined and structured interaction protocol; integrating the transport and social layer. A utility function is presented on the basis of individual and combined attributes. The agents negotiate on the type, location and the start time of social activity.

Hendrickson and Plank [11] studied the flexibility in trip departure times of the individuals focusing on fixed home-work trips. The authors developed a multinomial logit model to estimate the relation and significance of different attributes influencing choice of the transport mode and trip departure time. The authors proposed an equation to define the personal utility or preferences for a given set of departure times for the work trip. It is worth noting that the authors gave a special consideration to trip departure time characteristics in case of a shared transportation mode.

Knapen et al. [12] presents an automated, Global Car Pooling Matching Service (GCPMS), advisory service to match commuting trips for carpooling. The probability for successful negotiation is calculated by means of a learning mechanism. The matcher needs to deal with dynamically changing graph w.r.t. topology and edge weights. 


\section{Agent-based negotiation model}

The agent-based negotiation model for the long term carpooling is simulated to account for individual specific behavior during the carpooling process. The goal is to simulate the interactions of autonomous agents, to enable communication to trigger the negotiation process by incorporating a personalized preference function. The purpose is to introduce a behaviorally sound negotiation mechanism that determines the extent to which people need to adapt their daily schedule to enable cooperation and accommodate for a carpooling activity. The agents can interact with each other autonomously to find matching partners in order to co-travel in several different consecutive carpools; each of which corresponds to a multi-day period.

The procedure of negotiation and trip execution in the long-term carpooling can be broadly classified into three stages namely; (i) exploration and communication, (ii) negotiation, and (iii) carpooling (long-term trip execution).

In this paper, however, we focus on the proposed negotiation mechanism that efficiently represents the actual human preferential behavior based on a number of influencing factors. In order to make the negotiation mechanism, more accurate, a methodology is introduced to specified and personalized preference function. The proposed preference function for the selection of the most preferred trip departure time; partly derived from existing departure time studies is based on a number of factors namely; (i) travelling factors, (ii) socio-economic factors and (iii) time pressure factors.

For the departure time choices, we acquired the Hendrickson's multinomial logit model [11] for the work trips. The Hendrickson's base model included up to twenty eight alternatives, indicating combinations of four modes (drive alone, shared ride, transit with walk access and transit with auto access) and seven different departure time intervals of $10 \mathrm{~min}$ each. We use coefficients of the shared mode only, of the Hendrickson's multinomial logit model, for the carpooling trips. To make it continuous, we took different departure time intervals of one $\mathrm{min}$ instead of $10 \mathrm{~min}$. Apart from these departure time influencing factors, the driver and vehicle selection is based on the inspection of the individual's profiles (car and driving-license ownership).

In the simulation model, a "negotiation mechanism" is used to adapt the trip start times of an individual. The commuting trips in daily schedules (home-towork $H W$ and work-to-home $W H$ ) are considered. Home and work locations, trip start times $(H W$ and $W H)$ and their durations, and activity duration, the SEC attributes, including vehicle and driving-license ownership are used as input.

For the experiments described in this paper, the operational activity-based model for the region of Flanders (Belgium) FEATHERS [13] is used to generate a planned schedule for each member of the synthetic population. Mutually independent individuals using a transportation network free from unexpected congestion, are concerned. The initial daily plans are assumed to be optimal, i.e. generating maximal utility and hence to reflect the owner's preferences.

The negotiation and trip execution in the carpooling process is described in more detail in the following subsections. 


\subsection{Exploration and communication}

Each agent looks for other individuals to cooperate while executing its periodic trip by exploring the carpooling social network. People decide to select carpool partners from the group of individuals who share respectively the home and work locations with them. It is assumed that people board and alight at home and at work locations only. The framework is based on traffic flows between traffic analysis zones (TAZ) as opposed to specific street addresses.

The agents belonging to the same groups may communicate with each other by sending and receiving text messages. Through communication, the agents may negotiate on start time of the trips $(H W$ and $W H)$, on the vehicle to use and hence on the selection of the driver. If the agent decides to carpool, (s)he may start to explore for partners in the exploration phase, otherwise (s)he continues traveling solo. This agent may remain in the exploration phase throughout the simulation period (in case (s)he is unable to find a carpool partner).

The agent's behavior is modeled by a finite state machine. Each agent can send and/or receive messages to/from the other agents of the same group, as shown in fig. 1. The following messages are used: CarpoolRequestMessage, AcceptMessage and RejectMessage.

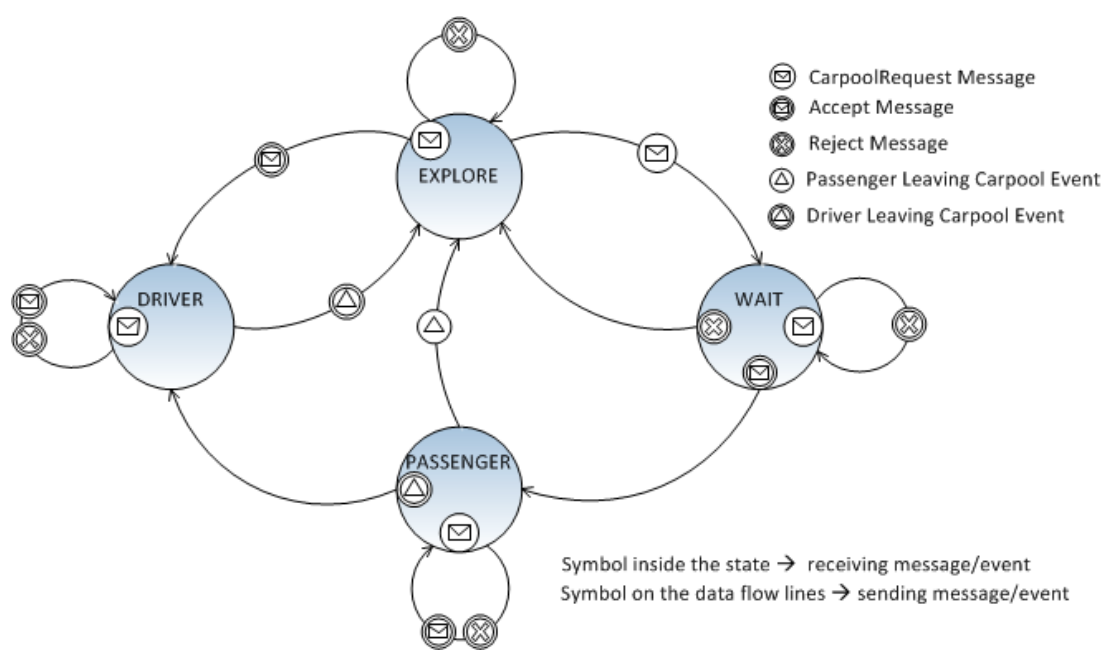

Figure 1: State-transition diagram of an agent $a_{i}$.

An agent performs the following activities in different states.

1. In the EXPLORE state, each agent (sender) may search for a partner (receiver) by sending a carpool invitation to a randomly chosen agent. For every simulated day, emission of invitations depends on the given probabilityToInvite parameter. As soon as an invitation has been emitted, the sender enters the WAIT state, waiting for the receiver's response. In the EXPLORE state, an agent can receive carpool invitations from other agents.

2. In the WAIT state, if the receiver's response is an AcceptMessage then the sender tries to join the CarPoolGroup the receiver belongs to and the sender 
changes its state to PASSENGER. If the response is a RejectMessage, the inviting agent changes its state to EXPLORE again in order to try to find a partner. In the WAIT state, any incoming invitation is rejected.

3. In the DRIVER state the agent plays the DriverRole in a CarPoolGroup, can receive carpool invitation and replies with either AcceptMessage or RejectMessage depending on the sender's departure time requirements and on the remaining car capacity. If the carpool period for the driver expires, then the agent will leave its DriverRole and change its state to EXPLORE.

4. In the PASSENGER state the agent continues to play the PassengerRole in the CarPoolGroup until the carpool period expires. While being a passenger, the agent handles carpool invitations in the same way as a driver.

Handling incoming invitations during the carpool lifetime, requires additional negotiation among the carpoolers and the new candidates to join the pool.

\subsection{Negotiation}

The matching is applied in the negotiation phase where final decisions to carpool are taken. The agents negotiate on trip $(H W$ and $W H)$ departure times and also about who will become the driver. The driver and vehicle selection is based on the inspection of the individual's profiles. The schedule adaptation depends on the preferences among feasible schedules of the individuals. The negotiation will become successful only when the individuals' preferred trip start times for both the trips $(H W$ and $W H)$ are mutually compatible within the carpool.

\subsubsection{Preference time function}

Several factors affect the preference function for the trip departure time of an agent. The travelling factors involved during the actual carpool trip execution are; (i) free flow travel time, (ii) expected congestion, (iii) waiting time and (iv) access time. The socio-economic factor (i.e. the ratio of travelling cost to annual income) helps to quantify the concept of value of time for departing at a particular time in the given time interval. The individuals' tolerance level for arriving late or early for a specific activity indicates the level of rigidity in the starting times of different activities.

In order to construct a behaviorally accurate model, Hendrickson's multinomial logit departure time choice model for work trips is used. People do not have a constant level of preference for every moment in the in the entire feasible time interval due to many factors such as time pressure.

The eqn (1) is used to determine the actual utility or satisfaction value of a particular agent to depart at a specific time in its available time window. The coefficients are taken from Hendrickson's study for the specific mode (shared transport). Consider $N$ agents $a_{1}, a_{2}, \ldots a_{N}$. The departure time $t_{1}, t_{2}, t_{3} \ldots, t_{T}$ available among the set of departure time $T$. The utility or preference $V_{a_{i} t_{j}}$ is specified to be 


$$
\begin{aligned}
V_{a_{i} t_{j}}=-2.09- & 0.008\left(F F T a_{i}\right)-0.021\left(\operatorname{CONG}_{t_{j}}\right)-0.699\left(\frac{\operatorname{COST}}{\operatorname{INCOME}}\right) a_{i} t_{j} \\
& -0.095\left(\text { ACC }_{a_{i} t_{j}}\right)-0.088\left(\text { WAIT }_{a_{i} t_{j}}\right)-0.148\left(\text { LATE }_{a_{i} t_{j}}\right) \\
& +0.0014\left(\operatorname{LATE}_{a_{i} t_{j}}\right)^{2}-0.01\left(\text { EARLY }_{a_{i} t_{j}}\right) \\
& -0.00042\left(\operatorname{EARLY}_{a_{i} t_{j}}\right)^{2}
\end{aligned}
$$

$V a_{i} t_{j}: \quad$ Utility/preference function of an agent for a particular time.

$P_{a_{i} t_{j}}: \quad \quad \quad \quad$ Probability to select a specific departure time.

FFTT $_{a_{i}}$ : $\quad$ Free flow Travel Time in carpool vehicle (i.e. $75 \%$ of travel time during peak time and $90 \%$ otherwise).

$\operatorname{CONG}_{t_{j}}$ : Portion of travel time associated with congestion (i.e. $25 \%$ of travel time during peak time and $10 \%$ otherwise).

$\left(\frac{\operatorname{COST}}{\operatorname{INCOME}}\right)_{a_{i} t_{j}}$ : Ratio of annual cost of carpooling to income level per annum. It depends on the time-of-day because toll and parking charges are included and those can be time-of-day dependent.

WAIT $_{a_{i} t_{j}}$ : Waiting time w.r.t. individual's most preferred time to depart.

ACC $_{a_{i} t_{j}}$ : Access time (we assume that the time required to pick/drop).

$\operatorname{LATE}_{a_{i} t_{j}}$ : Late arrival at work associated with the departure time.

$E A R L Y{ }_{a_{i} t_{j}}$ : Early arrival at work associated with the departure time. For the co-efficient of $E A R L Y_{a_{i} t_{j}}$ (i.e. we took 0.01) smaller magnitude than that of $L A T E_{a_{i} t_{j}}$; this is because late arrival at work is felt to be more onerous than early arrival.

The departure time choices are treated as simultaneous interactive decisions based upon maximization of individual travellers' utility or satisfaction with each departure time combination. The probability of an individual selecting departure time alternative $P_{a_{i} t_{j}}$ of the carpool is as in eqn (2);

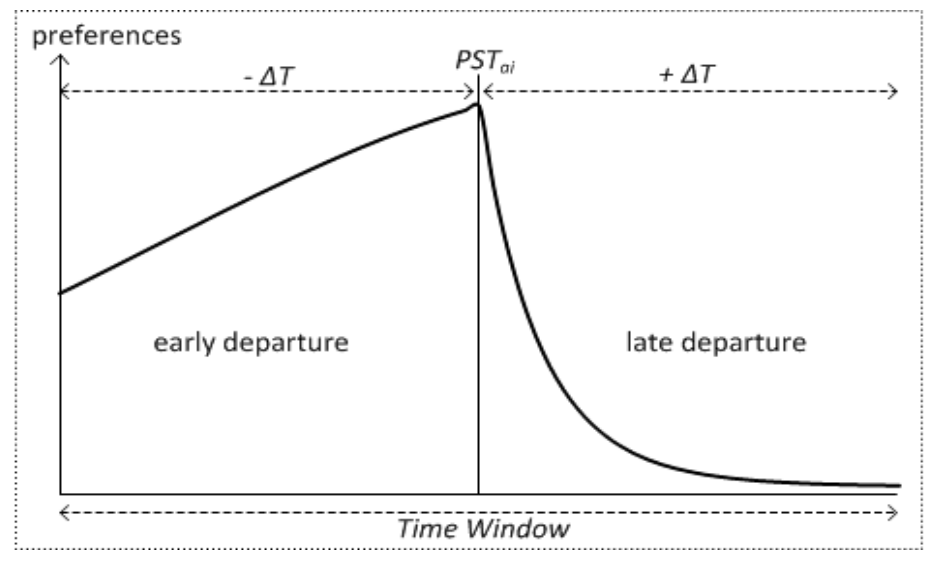

Figure 2: Departure time preference curve for an agent $a_{i}$. 


$$
P_{a_{i} t_{j}}=\frac{\exp \left(V_{a_{i} t_{j}}\right)}{\sum_{\mathrm{T}} \exp \left(V_{a_{i} \mathrm{~T}}\right)}
$$

The probability can be calculated for the discrete cases mentioned by Hendrickson. The results have been used to construct the continuous preference function shown in fig. 2. This was done because, for the simulation, we need to calculate the individual preference value for each possible trip start time in the candidate specific time window (e.g. the optimal time window $\pm \Delta t=30$ minutes).

\subsubsection{Negotiation for trip departure time}

After the assignment of an individual preference function based on the factors elaborated above for each agent, a negotiation mechanism could be employed in order to determine the carpool trip departure time.

For an agent $a_{i}$, the earliest and latest departure times for the trip are $T W_{L, a_{i}}$, $T W_{U, a_{i}}$ (lower and upper bounds for the time window). The preferred trip start time of $a_{i}$ is $P S T_{\text {Trip }, a_{i}}$.

In the simplest case, the individual is assumed to accept a symmetric deviation $\pm \Delta T$ w.r.t. the preferred trip start time. In general, this is not necessarily true since preceding or succeeding activities can induce timing constraints.

The possible lower and upper bounds for the trip of $a_{i}$ are given by eqn (3).

$$
\begin{aligned}
& T W_{L, a_{i}}=P S T_{{\text {Trip }, a_{i}}_{i}}-\Delta T \\
& T W_{U, a_{i}}=P S T_{{\text {Trip }, a_{i}}}+\Delta T
\end{aligned}
$$

The negotiation outcome is assumed to be associated to the intersection's length of the time intervals of the individuals. The following eqn (4) show the lower and upper bounds for the trip of the carpool; the indices used for the $\max ()$ function range over the set of candidate participants).

The available time intervals for the carpool are given by the eqn (4)

$$
\begin{aligned}
T W_{L, \text { carpool }} & =\max _{j=1 \ldots N}\left(T W_{L, j}\right) \\
T W_{U, \text { carpool }} & =\min _{j=1 \ldots N}\left(T W_{U, j}\right)
\end{aligned}
$$

The product of the sum of the probabilities of the departure time alternatives of the carpool participants for the intersection time intervals is

$$
P_{\text {carpool }}=\prod_{i=0}^{n} \sum_{j=T W_{L, \text { carpool }}}^{T W_{U, \text { carpool }}}\left(P_{a_{i} t_{j}}\right)
$$

The negotiation succeeds if and only if

$$
P_{\text {carpool }}>\text { prob of success }
$$

For every agent, the preference for a given time of departure is assumed to be proportional to the probability that the person will select that time.

$$
V_{a_{i} t_{j}}=k\left(P_{a_{i} t_{j}}\right)
$$


We assume that the combined preference for all carpoolers is the product of the preference values.

$$
V_{\text {carpool }, t_{j}}=\prod_{i \in \text { carpool }} k\left(V_{a_{i} t_{j}}\right)
$$

The effective trip start time $T S T_{\text {carpool }}$ of the carpool is given by

$$
T S T_{\text {carpool }}=\underset{j=T W_{L, \text { carpool to }} T W_{U, \text { carpool }}}{\arg \max }\left(V_{\text {carpool }, t_{j}}\right)
$$

For the evening $(W H)$ trip; we assume and took the probabilities of the departure time alternatives of the morning trip $(H W)$ but mirrored in time (see fig. 3).

In the simulation, for the start time of $H W$ and $W H$ trips, the negotiation succeed if and only if

$$
\begin{gathered}
\prod_{i=0}^{n} \sum_{j=T W_{H W L, \text { carpool }} \text { AND }}^{T W_{H W U, \text { carpool }}}\left(P_{a_{i} t_{j}}\right) \\
\prod_{i=0}^{n} \sum_{j=T W_{W H L, \text { carpool }}}^{T W_{W H U, \text { carpool }}}\left(P_{a_{i} t_{j}}\right)
\end{gathered}
$$

The effective trip start times of the carpooling trips $(H W$ and $W H)$ are given by eqn (11)

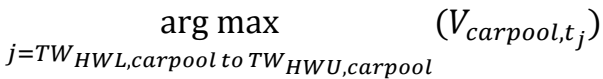

$$
\begin{aligned}
& \text { AND }
\end{aligned}
$$

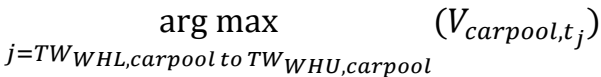

An individual decides to join the carpool if and only if the preferred trip start times for both the trips $(H W$ and $W H$ ) within the appropriate intervals.

After successful negotiation, the carpool participants adjust their schedule. The individual's resulting schedule applies to every working day during the period of carpooling.

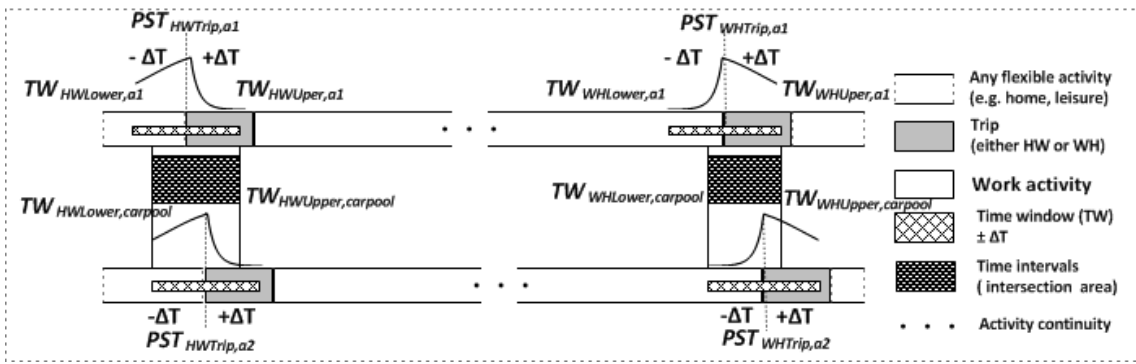

Figure 3: Negotiation on trip $(H W$ and $W H)$ departure times of two agents. 


\subsection{Carpooling (trip execution)}

The carpooling activity corresponds to the execution of the trips ( $H W$ and $W H$ ) over multiple days. The model assumes that travel times are insensitive to the level of carpooling (i.e. carpooling does not significantly decrease congestion). Travel times between locations have been computed a priori and are assumed to be time independent. This is to be refined by making the negotiation aware of time dependent travel time. The carpool candidates can explore for partners whenever needed.

\section{Simulation experiments and discussions}

The proposed model was run for data created by the FEATHERS activity-based model for the Flanders region. For the experiments, data for 30,000 individuals from a set of selected zones is used. An exploring individual is allowed to contact 5 other people at most during every simulated day. If the ProbabilityToInvite is $100 \%$ then (s)he must send carpooling requests. Otherwise, (s)he can decide not to emit any request. A carpooler determines the number of working days to carpool by selecting a number randomly from 30 to 60 . Obviously, a carpool is composed only if a driver is available. Four people at most can share a car (driver included).

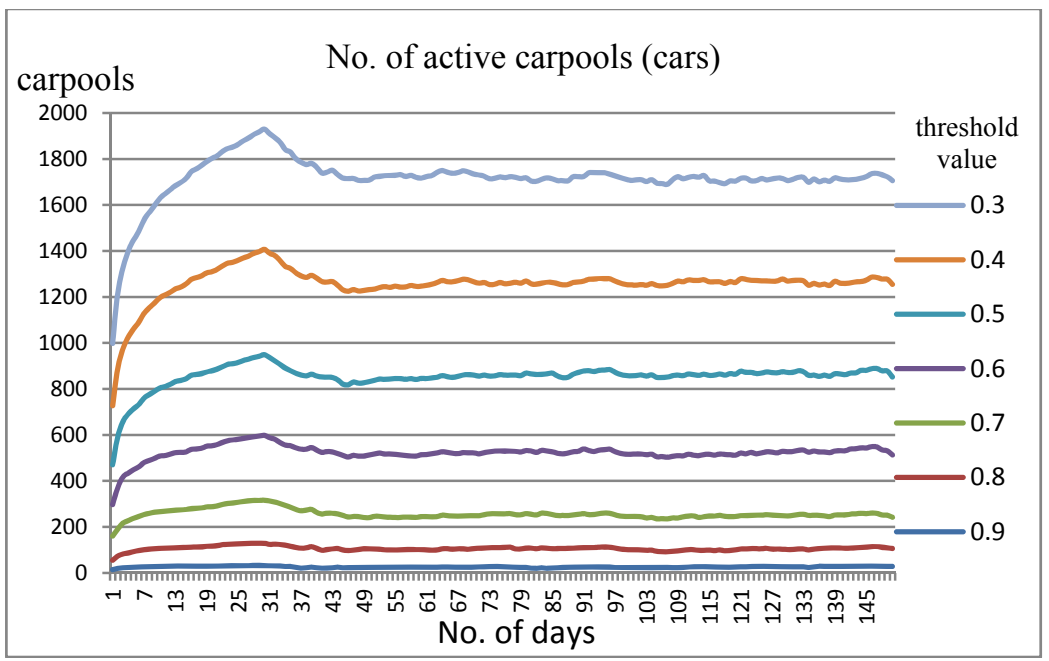

Figure 4: The number of active carpools of different threshold values.

Fig. 4 shows the results for a simulation where the individuals could adapt the trip start time with a specific threshold values i.e. $0.9,0.8,0.7,0.6,0.5,0.4$ and 0.3 were used. The line graph shows the number of active carpool groups over 150 working days. The horizontal axis shows the working days and the vertical axis represents the number of active carpool groups for each day. It is observed that on average, a lower threshold value allows for more carpooling. During the 
first 30 days the number of groups monotonically increases since the shortest possible carpooling period lasts for 30 days. After 30 days, the curves show a decrease because new carpoolers seem to join existing groups rather than create new ones. It seems to be easier to join an existing group than to create a new one: the number of carpools decreases but the number of participants does not decrease in that period. A curves remain same with slightly increase and remains same for the number of carpool groups after 45 days because the possibility to join existing carpool group is same as the creation of the new carpool groups.

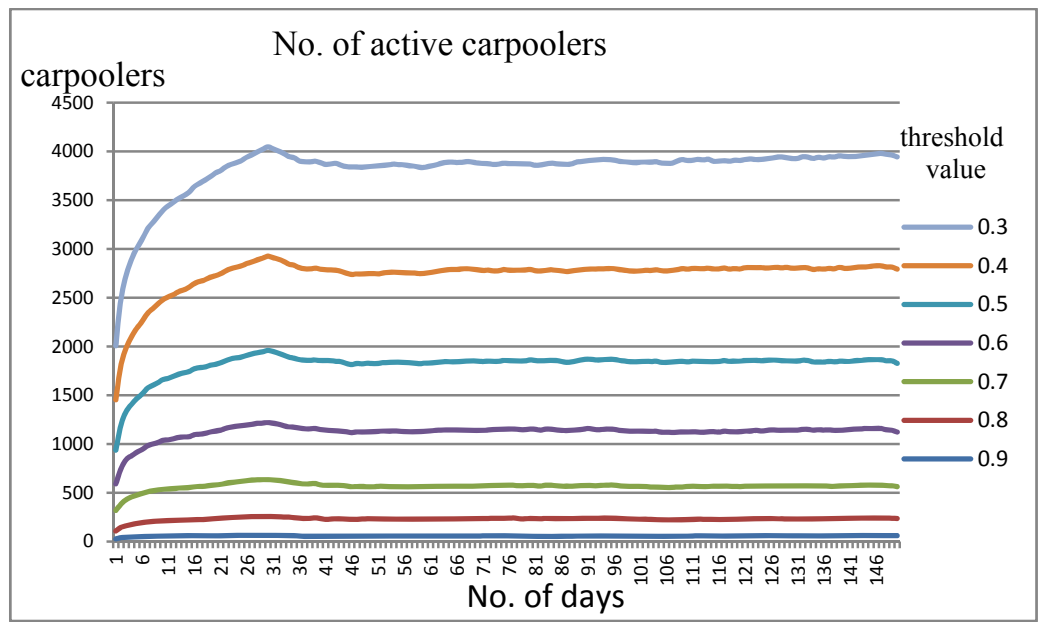

Figure 5: The number of active carpoolers for different threshold values.

The graph in fig. 5 shows the number of active carpoolers throughout the simulation period. For each threshold value, the number of active carpoolers rapidly increases at the start of the simulation up to about 30 days. After 30 days, the increase rate is lower up to the end of the simulation.

\section{Conclusion and future work}

Modeling the interaction between individual agents becomes progressively important in recent research. An agent-based framework using the Janus organization concept has been setup to evaluate the evolution of a carpooling society under several conditions. The model aims to analyze various effects of agent interaction and behavior adaptation. This paper covers the concept of communication, negotiation and coordination for the long term carpooling of a multiple trip model. The agents negotiate on trip (morning and evening) departure times and on the driver assignment. During the negotiation process the agents may adapt their daily schedules to enable cooperation. The data used for implementation have been created by the FEATHERS activity-based model for the Flanders region. The results show that when the threshold value is lower, 
the chances for negotiation success are greater. The simulation model on the Janus platform provides a solution to the complex problems of mutual adaptation.

The future research will mainly focus on incorporating different daily activities in addition to work and home activities. More elaborated schedule adaptation will be integrated in the model.

\section{References}

[1] Galland S, Knapen L, Yasar A, Gaud N, Janssens D, Lamotte O, Koukam A, Wets G. Multi-agent simulation of individual mobility behavior in carpooling. TRC, 2014; 45: 83-98.

[2] Bellemans T, Bothe S, Cho S, Giannotti F, Janssens D, Knapen L, Koerner C, May M, Nanni M, Pedreschi D, Stange H, Trasarti R, Yasar A, Wets G. An Agent-Based model to evaluate carpooling at large manufacturing plants. Procedia Computer Science, Niagara. 2012.

[3] Kamar E and Horvitz E. Collaboration and shared plans in the open world: Studies of ridesharing. The Twenty-First International Joint Conference on Artificial Intelligence, 2009.

[4] Hussain I, Knapen L, Bellemans T, Janssens D, Wets G. An agent-based negotiation model for carpooling: A case study for Flanders (Belgium), $94^{\text {th }}$ Transportation Research Board, 2015.

[5] Cossentino M, Gaud N, Hilaire V, Galland S, Koukam A. ASPECS: an agent-oriented software process for engineering complex systems - how to design agent societies under a holonic perspective, Autonomous Agents and Multi-Agent Systems. 2010; 20: 260-304.

[6] Gaud N, Galland S, Hilaire V, Koukam A. An organizational platform for holonic and multiagent systems, $7^{\text {th }}$ International Conference on Autonomous agents \& Multiagent Systems, Portugal. 2008; 111-126.

[7] Horvitz E, Apacible J, Sarin R, Liao L, Prediction, expectation, and surprise: Methods, designs, and study of a deployed traffic forecasting service. UAI, AUAI Press, 2005; 275-283.

[8] Rady HA. Multi-Agent System for Negotiation in a Collaborative Supply Chain Management. International Journal of Video \& Image Processing and Network Security. 2011. 11(5).

[9] Hussain I, Knapen L, Galland S, Janssens D, Bellemans T, Yasar A, Wets G. Organizational and agent-based automated negotiation model for carpooling, EUSPN'14, 2014.

[10] N. Ronald, T. Arentze, H. Timmermans, An agent-based framework for modelling social influence on travel behaviour, Proceedings of the $18^{\text {th }}$ World IMACS Congress and MODSIM09 International Congress on Modelling and Simulation, 2009.

[11] Hendrickson C, and Plank E, The Flexibility of Departure Times for Work Trips, Transportation Research Part A, 18(1), 25-36, 1984. 
[12] Knapen L, Yasar A, Cho S, Keren D, Abu Dbai A, Bellemans T, Janssens D, Wets G, Schuster A, Sharfman I, Bhaduri K. Exploiting Graphtheoretic Tools for Matching in Carpooling Applications. JAIHC. 2013; $17 \mathrm{p}$.

[13] Bellemans T, Kochan B, Janssens D, Wets G, Arentze T, Timmermans H., Implementation framework and development trajectory of feathers activity-based simulation platform. Transport. Research Record: J., 2010, 111-119. 